\title{
Die Kollektivierung der Norm und kollektive Normen
}

\author{
Inês Fernandes Godinho
}

\section{Einleitung ${ }^{1}$}

Das Thema der Kollektivierung setzt, insbesondere wenn eine Ausländerin versucht, dazu etwas Logisches zu sagen, eine Verständigung über den Begriff voraus.

In einem Rechtslexikon ${ }^{2}$ steht der Begriff nicht, also handelt es sich wohl nicht um einen Rechtsbegriff. Suchen wir in einem allgemeinen Wörterbuch ${ }^{3}$, finden wir zwei Deutungen: die ältere, traditionelle, und die alltägliche, »normale«. Der normale Gebrauch des Wortes meint die Überführung privater Produktionsmittel in die Gemeinwirtschaft, üblicherweise im landwirtschaftlichen Kontext. Für unser Thema in einem juristischen Arbeitskreis fällt es allerdings schwer, dies als die ausschlaggebende Bedeutung zu akzeptieren, was uns dann zu der zweiten, vom lateinischen collectivus abstammenden Bedeutung führt, nämlich der des organisierten Zusammenschlusses von Menschen zu Gemeinschaften, Vereinen oder Genossenschaften. Mit dieser Bedeutung werde ich im Folgenden arbeiten.

Dieser organisierte Zusammenschluss muss sich dennoch im Rahmen des Themas als eine Herausforderung für das Strafrecht darstellen, besonders aus der Perspektive der Normentheorie. Es stellt sich hier keine einfache Aufgabe, deswegen nähere ich mich ihr in mehreren Schritten. Auf der einen Seite wird das Problem der Kollektivierung der Norm behandelt, nämlich die Frage, inwiefern dieser Zusammenschluss von Menschen zu Gemeinschaften etwas mit dem Individuationsprozess bzw. Entstehungsprozess der Norm zu tun hat. Auf der anderen Seite wenden wir uns

1 Ich danke Prof. Dr. Petra Weber für die Revision der ersten deutschen Fassung und Prof. Dr. Joachim Renzikowski für die zweite Revision und kritische Anmerkungen.

2 Z.B. Creifelds, Rechtswörterbuch, 23. Aufl. 2019 oder Geiger, Beck'sches Rechtslexikon, 3. Aufl. 2003.

3 Z.B. Duden-online „Kollektivierung“, abrufbar unter https://www.duden.de/rechts chreibung/Kollektivierung (letzter Zugriff am 21.08.2020); Wikipedia „Kollektivierung“, Version vom 13.2.2020, 10.19 Uhr, abrufbar unter https://de.wikipedia.org/ wiki/Kollektivierung (letzter Zugriff am 21.08.2020). 
der kollektiven Norm zu und darüber hinaus der Frage, was eine solche Norm ist. Die Verknüpfung beider Aspekte wird dann in einem dritten Schritt analysiert, indem ich die Kollektivierung der Eigenschaft des Kollektivs gegenüberstelle.

\section{Kollektivierung der Norm}

Die Frage der Kollektivierung der Norm hängt, im Rahmen dieses Beitrags, mit dem Entstehungsprozess und folglich mit dem Individuationsprozess der Norm zusammen. Ich gehe von der Voraussetzung aus, dass Normen als Folge des organisierten Zusammenschlusses von Menschen zu Gemeinschaften betrachtet werden können und dass es ohne diesen $\mathrm{Zu}$ sammenschluss keine Normen geben kann. Eigentlich handelt es sich um die Grundlage der Norm, d.h. nicht um ihren Sinn (was sie ist), sondern um ihren Grund (warum sie ist). Das Problem kann auch als Frage formuliert werden: Könnte es »echte" Normen geben, wenn es nur eine Person gäbe? In dem Film Cast Away - Verschollen (USA, 2000; mit Tom Hanks) befindet sich der Charakter Chuck Noland auf einer Insel und versteht, dass er dort allein ist. Recht schnell sucht er die Gesellschaft eines imaginären Freundes, in Gestalt eines Volleyballs namens Wilson. Noland kann sich nicht als komplett allein verstehen (trotz des Wissens, dass Wilson lediglich ein Volleyball ist). Ergibt es in einer solchen Situation - wenn ein Mensch vollständig für sich allein ist - überhaupt Sinn, von (rechtlichen) Normen zu sprechen? Es herrscht wohl Einigkeit darüber, »dass das Recht eine Ordnung menschlichen Handelns ist, die das Zusammenleben regelt«. ${ }^{4}$ Wenn das Recht als Koordination menschlichen Zusammenlebens betrachtet wird, fällt die Möglichkeit aus, dass eine Norm nur für einen einzigen Menschen existiert. Die Norm ist, weil es einen Zusammenschluss von Menschen zu einer Gemeinschaft gibt (auf die konkrete Form der Gemeinschaft kommt es nicht an).

Ohne näher auf die Diskussion über Normen als Bestimmungsnormen oder Bewertungsnormen einzugehen ${ }^{5}$, zumal auch solche eine Mehrzahl von Personen voraussetzen, kann es eine strafrechtliche Tat nur geben, wenn sie als solche in einer Norm in der Form eines Gesetzes vorgesehen

4 Zippelius, Rechtsphilosophie, 6. Aufl. 2011, S. 3.

5 Renzikowski, in: Borowski/Paulson/Sieckmann (Hrsg.), Rechtsphilosophie und Grundrechtstheorie, 2017, S. 631 (635 ff.). 
ist (Gesetzlichkeitsprinzip ${ }^{6}$ ). Die gesetzliche Norm spiegelt einen Willen wider. Dennoch ist nicht jeder Wille relevant für die Entstehung der Norm. Strafrechtliche Normen können nicht von einem einzelnen Bürger als solche ausgeschrieben oder genehmigt werden. Wenn eine Norm als strafrechtliches Verbot gelten soll, kann es nicht der einfache Bürger sein, der sie vorschreibt. Vielmehr gründen sich strafrechtliche Normen auf einer bestimmten staatlichen auctoritas. ${ }^{7}$ Auch wenn die Norm einem Willensakt entspricht - schon Kelsen sagte, die Norm sei der Sinn eines menschlichen Willensaktes (auf ein Sollen gerichtet) ${ }^{8}-$, heißt das nicht, dass sie nur den Eigenwillen eines einzelnen Menschen darstellt. ${ }^{9}$ Dieser Willensakt ist mehr die voluntas einer institutionellen Macht. M.a.W.: Nur nach einem Zusammenschluss von Menschen zu Gemeinschaften kann ein Bedarf an Normen entstehen. Dieser Bedarf wird dann vom Individuum anerkannt und die Institution (auctoritas) des Normgebers versorgt die Individuen bedarfsgerecht. Wie Raz formuliert:

A person has authority either if he is regarded by others as having authority or if he should be so regarded. ${ }^{10}$

Gesellschaftliche Kollektivierung ist also ein Bestandteil des Individuationsprozesses einer Norm. Wenn ich zum Beispiel allein wohne und zu mir selber sage - vielleicht sogar auf ein Blatt Papier schreibe -, dass ich jeden

6 Vgl. Kindhäuser/Zimmermann, Strafrecht, Allgemeiner Teil, 9. Aufl. 2020, 33 Rn. 1 ff.; Jescheck/Weigend, Strafrecht, Allgemeiner Teil, 5. Aufl. 1996, S. 131 ff.; Dias, Direito Penal, Tomo I, 3. Aufl. 2019, S. 209 ff.; Costa, Revista de Legislação e de Jurisprudência 2002, 354 ff.; Godinho, in: Sieber/Jarvers/Silverman (Eds.), National Criminal Law in a Comparative Legal Context, Vol. 2.2. 2017, S. 53 ff.; Palaz$z o$, Legalità penale. Considerazioni su transformazione e complessità di um principio 'fondamentale', Quaderni Fiorentini per la Storia del Pensiero Giuridico Moderno 26, 2007, S. 1279 (1282 ff.).

7 Es handelt sich hier um auctoritas als eine Ordnungsgrundlage; auf die Diskussion über die Unterscheidung zwischen auctoritas/potestas wird hier spezifisch nicht eingegangen. Näher dazu Kessel, ARSP 1959, 215 (224 ff.).

8 Laut Kelsen, Reine Rechtslehre, 2. Aufl. 1960, S. 27: "Das ist der Sinn, den gewisse menschliche Akte haben, die intentional auf das Verhalten anderer gerichtet sind. Sie sind intentional auf das Verhalten anderer gerichtet, wenn sie, ihrem Sinne nach, dieses Verhalten gebieten (befehlen), aber auch wenn sie es erlauben und insbesondere wenn sie es ermächtigen, das heißt: wenn dem anderen eine gewisse Macht verliehen wird, insbesondere die Macht, selbst Normen zu setzen. Es sind - in diesem Sinne verstanden - Willensakte".

9 Ausgenommen, bei Kelsen, wenn es sich um eine richterliche Entscheidung handelt.

10 Raz, Practical Reasons and Norms, 1999, S. 62 f. 
Tag die Küche aufräumen soll, ist das keine Norm. Auch wenn damit ein Sollen gemeint ist - die Küche soll aufgeräumt werden - ist dieses individuelle Sollen kein normatives Sollen. ${ }^{11}$ Wir kennzeichnen eine Norm als solche, indem wir sie erkennen, erstens, weil sie uns bekannt gemacht worden ist, und zweitens, weil die Institution, die sie gesetzt hat, dazu legitimiert ist. Zum Beispiel werden die Regularien einer Universität befolgt, weil Professoren und Studenten wissen, dass diese Normen von den entsprechenden Organen - in akademischen Beratungen usw. - erlassen wurden. Auch die Moralnormen entstehen nicht, weil eine Person sich plötzlich eine Norm ausgedacht hat. Die Normen der Moral - trotz aller Unterschiede zu gesetzlichen Normen - setzen auch eine Kollektivierung in ihrem Entstehungsprozess voraus:

Den Ausdruck, Kulturnormen' gebrauche ich als einen Sammelnamen für die Gesamtheit derjenigen Gebote und Verbote, die als religiöse, moralische, konventionelle, als Forderungen des Verkehrs und des Berufs an das Individuum herantreten. ${ }^{12}$

Die Frage ist dann, auf welche Gemeinschaften sich Normen beziehen. Gemeinschaften gab es schon immer. Das Modell von (rechtlichen) Gemeinschaften, die durch Individuen und Vertragsbeziehungen konstituiert waren, ${ }^{13}$ hat sich seit der Aufklärung entwickelt in einer Auffassung, die menschliche Verbände als lebendige Ganzheiten betrachtet:

Im Zeitalter des Individualismus und des Rationalismus wollte man die Gesellschaft aus Individuen und Vertragsbeziehungen konstituieren: So führten die Lehren von einem Gesellschafts- oder Herrschaftsvertrag die Entstehung von Gesellschaft und Staat (...) auf einen freien Konsens zwischen den Einzelnen zurück. In der folgenden Zeit wandten sich aber die Organismustheorien gegen diesen Individualismus der Aufklärung: Die menschlichen Verbände seien nicht auf einzelne Individuen und bloß vertragliche oder vertragsähnliche Beziehungen zurückführbar. Vielmehr seien sie lebendige Ganzheiten, in denen, wie in einem lebendigen Organismus, das Ganze und die Teile aufeinander angewiesen seien. ${ }^{14}$

11 Demgegenüber anerkennt von Wright, Norm und Handlung. Eine logische Untersuchung, 1979, S. 85, neben heteronomen auch autonome Normen, weil er allein auf die Autorität des Normgebers abstellt: „Die Idee autonomer Normen lässt sich aber auch so verstehen, dass man diejenigen Vorschriften als autonom ansieht, die man sich selbst gibt.".

12 Meyer, Rechtsnormen und Kulturnormen, 1903, S. 17.

13 Man braucht hier nur an Rousseaus Gesellschaftsvertrag zu denken.

14 Zippelius (Fn. 4), S. 155. 
Dagegen dachte Kelsen, es gebe kein überindividuelles reales Substrat einer Gemeinschaft; die Frage sei vielmehr diejenige nach einem Einheit stiftenden Faktor in der Gemeinschaft, und dieser sei die Ordnung:

Die Gesellschaft als Gegenstand einer normativen Gesellschaftswissenschaft ist eine normative Ordnung des gegenseitigen Verhaltens der Menschen. (...) Wenn man sagt, daß eine bestimmte Gesellschaft durch eine normative, das gegenseitige Verhalten einer Vielheit von Menschen regelnden Ordnung konstituiert ist, so muss man sich bewußt bleiben, daß Ordnung und Gesellschaft nicht zwei voneinander verschiedene Dinge, sondern ein und dasselbe Ding sind, daß die Gesellschaft in nichts anderem besteht als in dieser Ordnung, und daß, wenn Gesellschaft als Gemeinschaft bezeichnet wird, im wesentlichen die das gegenseitige Verhalten der Menschen regelnde Ordnung das diesen Menschen Gemeinsame ist. ${ }^{15}$

In einem Beispiel von Zippelius:

Ein Konzert kommt dadurch zustande, dass sich alle Musikanten an der Komposition, mithin an dem objektiven Ordnungsplan orientieren, den sie im Notenblatt vor sich liegen haben, so dass man (...) sagen könnte, das eigentlich Konstitutive im Konzert sei die Komposition. ${ }^{16}$

Diese Perspektive bringt uns aber nicht weiter. Wenn wir uns fragen, was die Gemeinschaft ist, und wenn die Gemeinschaft die Ordnung sein soll, erhalten wir keine Antwort auf die Frage, wer - um beim Beispiel zu bleiben - denn dann der Komponist ist. Wenn die Norm konstitutiv für die Gemeinschaft ist, wie kommt es zur konstitutiven Norm? Diese Frage versuchte Kelsen durch die hypothetische »Grundnorm « zu beantworten. ${ }^{17}$ Jedoch löst diese das Problem ebenso wenig. Die Norm kann ihrer Ordnungsfunktion genügen, wenn das zu Ordnende die Norm erkennt. Die Norm kann ihre Funktion als (Grund)Bestandteil einer Gemeinschaft nur erfüllen, wenn sie erstens nachvollziehbar ist und zweitens als »legitim» anerkannt wird. Auch wenn Normen als Handlungsmuster verstanden werden - egal ob rechtliche, soziale oder sittliche -, taugen sie nur dann als Muster, wenn ihr Autor als legitim anerkannt ist. Deshalb gelten etwa Ordnungswidrigkeitengesetze nicht supranational. Noch einfacher ist das

15 Kelsen (Fn. 8), S. 169 f.

16 Zippelius (Fn. 4), S. 156.

17 Kelsen (Fn. 8), S. 196 ff. 
Beispiel der »Höflichkeits«-Normen: Ein portugiesischer Tourist, der sich erstmalig in China befindet, »kann" einen Chinesen nicht über chinesische Höflichkeitsregeln belehren - das wird nicht ernst genommen. Umgekehrt jedoch kann ein Chinese dem portugiesischen Touristen sagen, dass in China etwas so oder so gemacht werden soll.

Der "Normgeber" muss also auctoritas haben, eine Legitimität, die Norm zu setzen, ansonsten handelt es sich lediglich um einen (unverbindlichen) Hinweis - und eben keine Norm - für die Gemeinschaft.

Graphisch könnte man es so darstellen:

$\mathrm{K}_{(\mathrm{ZG})} \rightarrow \mathrm{BN} \rightarrow \mathrm{AL} \rightarrow \mathrm{NG} \rightarrow \mathrm{N} \rightarrow \mathrm{K}$, wobei:

$K_{(Z G)} \quad$ die Kollektivierung (als Zusammenschluss zur Gemeinschaft);

$B N \quad$ der Bedarf einer Norm;

$A L \quad$ Akzeptierte Legitimität (auctoritas);

NG der Normgeber;

$N \quad$ die Norm;

$K \quad$ das Kollektiv ist.

\section{Kollektive Normen}

Damit überhaupt zwischen ,Kollektivierung' und ,kollektiv' unterschieden werden kann, ist es wichtig, nochmals einen Schritt zurück zu gehen, nämlich zu der Bedeutung von ,kollektiv'. Auch hier sind zwei Bedeutungen zu betrachten: ${ }^{18}$,kollektiv' i.S.v. ,gemeinschaftlich' oder i.S.v. , alle Beteiligten betreffend, umfassend'. Die erste Bedeutung führt uns zu einem ganz ähnlichen Blickwinkel wie im vorherigen Punkt. Das bringt uns nicht weiter, soll aber nicht vollends außer Acht gelassen werden. Interessanter für uns ist aber die zweite Bedeutung, alle Beteiligten betreffend‘.

Oben haben wir die Kollektivierung als Bestandteil des Individuationsprozesses der Norm unter Berufung auf die auctoritas des Normgebers herausgearbeitet. Die jetzt eingeführte zweite Bedeutung des Begriffs des Kollektivs führt uns nun nicht zur Institutionalisierung der Norm, einer theoretischen Dimension der Norm, sondern mehr zu deren Anwendung, also zu einer praktischen Dimension der Norm.

18 Duden-online „kollektiv“, abrufbar unter https://www.duden.de/rechtschreibung/ kollektiv (letzter Zugriff am 21.08.2020). 
Die praktische Dimension der Norm betrifft deren Adressaten - es geht um alle Beteiligten (der Gemeinschaft). Es handelt sich um ein Problem, das in zweierlei Hinsicht betrachtet werden kann: 1) Wer sind die Adressaten? 2) »Was« sind die Adressaten?

Schon in der graphischen Darstellung des Individuationsprozesses der Norm war als letzter Schritt das Kollektiv angedeutet. Dieser Prozess dient also der Ordnung der Gemeinschaft. Dabei können in mehreren Formen des Zusammenschlusses zu Gemeinschaften Entstehungsprozesse und Individuationsprozesse von Normen beobachtet werden, zuerst sicher im Staat, aber auch etwa bei juristischen Personen (z.B. die Satzung der Aktiengesellschaft). Das Kollektiv hat dann unterschiedliche Bedeutungen: Die Adressatengruppen - nämlich die verschiedenen Beteiligten - können sogar gegeneinanderstehen. Nur im Fall von staatlichen Gesetzen umfasst die Adressatengruppe unterschiedliche (kleinere) Gemeinschaften. Nicht alle staatlich gesetzten Normen richten sich an alle Gemeinschaften, weil sich trotz ihres abstrakt-generellen Charakters manche Gesetze nur an bestimmte Gruppen (z.B. Soldaten, Amtsträger) richten.

Im Fall von strafrechtlichen Normen handelt es sich um ein general-kollektives Handlungsmuster: Jeder Adressat soll nicht töten, unabhängig davon, auf welchem Gebiet er oder sie tätig ist (Professoren, Geschäftsleute, Wissenschaftler, Taxifahrer oder Unternehmen bei Rechtssystemen, in denen societas delinquere potest ${ }^{19}$ anerkannt ist).

Die Frage bei general-kollektiven Handlungsmustern wie strafrechtlichen Normen lautet: »Was« sind die Adressaten? In manchen Ländern sind die Normadressaten immer noch nur natürliche Personen. Das ist nicht mehr der Fall in Portugal, wo juristische Personen Adressaten des generalkollektiven Handlungsmusters sind (art. 11 [2] port. StGB). ${ }^{20}$ Juristische Personen sind also auch Zurechnungssubjekte, die für ihre Handlungen haften (verantwortlich gemacht werden).

Das führt uns zu der nächsten Frage: Was ist die Voraussetzung dafür, Adressat einer (Zurechnungs-)Norm zu sein? Adressat einer Norm - die ein Sollen darstellt - zu sein setzt voraus, dass dem Adressaten ein Normverstoß auch zugerechnet werden kann. Die Zurechnung eines Normverstoßes setzt die Möglichkeit einer Wahl zwischen zwei alternativen Hand-

19 Siehe u.a. Busch, Grundfragen der strafrechtlichen Verantwortlichkeit der Verbände, 1933; Schmitt, Strafrechtliche Maßnahmen gegen Verbände, 1958, S. 178 ff.; Hirsch, ZStW 107 (1995), 288 ff.; Dannecker, GA 2001, $101 \mathrm{ff}$.

$20 \mathrm{Zu}$ dieser Frage, in Portugal, Brito, in: Direito Penal Económico e Financeiro, 2012, S. 201 ff.; Costa, in: IDPEE, Direito Penal Económico e Europeu: Textos Doutrinários, Vol. I. 1998, S. $501 \mathrm{ff}$. 
lungen voraus. Ohne tiefer auf die Diskussion einzugehen, haben beide, natürliche wie juristische Personen, diese Möglichkeit. ${ }^{21}$ In den Worten von Renzikowski:

[E]in aktives Vermögen, ein bestimmtes Ereignis hervorzubringen, reicht noch nicht aus, um einen Normadressaten vollständig zu definieren. (...) Vielmehr ist ein weiteres Vermögen erforderlich, nämlich seinen Willen nach einer Maxime bestimmen zu können, oder auch: reflexives Bewusstsein. (...) Zurechnungsfähigkeit - oder auch Willensfreiheit - ist also die logische Voraussetzung, um überhaupt von einem Sollen und damit von Normen sprechen zu können. ${ }^{22}$

Heute wird im Hinblick auf Künstliche Intelligenzen vermehrt darüber diskutiert, ob »intelligente « Roboter - wie etwa Sophia von Hansen Robotics - strafrechtlich verantwortlich gemacht werden sollen, wenn sie »töten«. Diese Roboter lernen, und es kann schon gesagt werden, dass sie sehr bald »Entscheidungen« werden treffen können. Wie Gless/Seelmann es darstellen:

Denn heute illustriert der Einsatz von Robotern in immer mehr Lebensbereichen, dass das Recht überdacht werden muss, weil es nur unzureichend auf den Einsatz autonom agierender Maschinen eingerichtet ist, diese aber in erstaunlichem Tempo Tätigkeiten übernehmen, die zuvor nur Menschen übertragen war. Wer trägt die Verantwortung, wenn ein autonom fahrendes Fahrzeug die aus der Umgebung aufgenommenen Daten falsch interpretiert und dadurch etwa ein Hindernis nicht erkennt und infolgedessen einen tödlichen Auffahrunfall verursacht? ${ }^{23}$

Können solche Roboter auch Adressaten von general-kollektiven Handlungsmustern wie Strafrechtsnormen sein? Bisher war die Rolle des Normadressaten exklusiv an menschliche Adressaten gerichtet, die Mitwirkende am Rechtssystem sind. Um Adressat im Strafrechtssystem zu sein, muss dieser Adressat den objektiven Tatbestand erfüllen, ${ }^{24}$ also eine (strafrechtlich relevante) Tat hervorbringen können. Dafür müssen diese Adressaten von Normen ansprechbar sein, d.h., ein Sollen muss im Dialog Norm-

21 S. Aichele, PJRE 2008, 3 ff.; ders., in: Kühl (Hrsg.), Zur Kompetenz der Rechtsphilosophie in Rechtsfragen, 2011, S. $31 \mathrm{ff}$.

22 Renzikowski, in: Borowski/Paulson/Sieckmann (Hrsg.) (Fn. 5), S. 631 (638 f.).

23 Gless/Seelmann, in: Gless/Seelmann (Hrsg.), Intelligente Agenten und das Recht, 2016, S. 11 (12).

24 Renzikowski, in: Borowski/Paulson/Sieckmann (Hrsg.) (Fn. 5), S. 631 (639). 
Adressat möglich sein und Sinn ergeben. Es stellt sich daher die Frage: Auch wenn diese Agenten - wie Sophia - ,intelligent' sein und ,Entscheidungen' treffen können, kann man sie ohne Weiteres mit menschlichen Agenten gleichsetzen? Dazu Seher:

Die Antwort auf diese Frage beginnt mit einer normtheoretischen Überlegung: Welchem Zweck dient das Recht, und auf welche Weise will das Recht diesen Zweck erreichen? - Das Recht will als staatliches Normensystem das Leben derjenigen, die ihm unterworfen sind, auf verlässliche Weise gestalten und dadurch bestimmte Werte - etwa Freiheit, Sicherheit, Frieden oder Wohlstand - verwirklichen. Diese Zwecke verfolgt es durch Verhaltensbefehle und durch die Anordnung bestimmter Folgen für die Befolgung oder Missachtung dieser Befehle. ${ }^{25}$

Anhand der Idee der Kollektivierung, die oben vorgestellt wurde, ist die Antwort bezüglich dieser »intelligenten « Agenten meines Erachtens negativ. Normadressaten sind - auch wenn nur mittelbar - unabdingbar Teil des Zusammenschlusses zu einer Gemeinschaft, d.h. des Individuationsprozesses der Norm. Wirklichkeiten wie "Sophia« entstehen hingegen als einzelne, insbesondere bilden sie - solange sie das "Produkt « menschlicher Tätigkeit bzw. Herstellung sind - keine eigenen Gemeinschaften. Mit anderen Worten: Die Norm kann nur die Mitglieder einer Gemeinschaft als Adressaten haben. Damit wird auch der normative Gehalt von faktischen Tatsachen verstanden: Wenn die Normadressatengemeinschaft die Norm nicht mehr erkennt, ergibt sich der Bedarf nach (anderen) Normen, die dem Zusammenschluss der Mitglieder entspricht.

Graphisch könnte man es so darstellen:

$$
\mathrm{K} \rightarrow \mathrm{NA} \rightarrow \mathrm{NAK} \rightarrow \mathrm{K}_{(\mathrm{ZG})} \text {, wobei: }
$$

$K_{(Z G)} \quad$ die Kollektivierung (als Zusammenschluss zur Gemeinschaft);

NA der Normadressat;

Nak Normakzeptanz;

$K \quad$ das Kollektiv ist.

25 Seher, in: Gless/Seelmann (Hrsg.) (Fn. 23), S. 45 (47); s. auch Kindhäuser, Gefährdung als Straftat, 1989, S. 137 ff.; Renzikowski, Restriktiver Täterbegriff und fahrlässige Beteiligung, 1997, S. 57. 


\section{Kollektivierung und Kollektiv: Herausforderungen für das Strafrecht}

In der Wechselwirkung von Kollektivierung und Kollektiv - nach hiesigem Verständnis - ergeben sich einige Herausforderungen für das Strafrecht.

Die erste Herausforderung ist die normative Kraft von Tatsachen. Im Rahmen der Anerkennung der Norm durch die Gemeinschaft muss es eine Kongruenz zwischen dem Handlungsmuster der Norm und dem Grundsatz des Zusammenschlusses geben, damit die Norm als gültig für die Kollektive gelten kann; es muss also auch eine Konvergenz zwischen dem Handlungsmuster der Gemeinschaft und dem Handlungsmuster der Norm geben.

Die zweite Herausforderung besteht bezüglich der theoretischen Dimension der Norm, insbesondere der aus der Kollektivierung resultierenden auctoritas. In der Tat, die fragmentarische Natur der Gesellschaft unserer Zeit führt dazu, dass viele kleinere Gemeinschaften in der Gesellschaft anzutreffen sind. Hieraus ergibt sich, dass die auctoritas des Normgebers nicht nur auf dem Niveau des Staates anerkannt wird (Gesetzgebung), sondern auch in anderen Wirklichkeiten. Beispiel: Juristische Personen haben heute die auctoritas, (durch ihre Organe) Normen zu erlassen, insbesondere im Rahmen von Compliance-Normen. Das bedeutet, dass diese - nicht gesetzlichen - Normen in der kleineren Gemeinschaft des Unternehmens gelten. Die schon erkannte Herausforderung besteht darin zu wissen, wie verbindliche Normen von unterschiedlichen auctoritates miteinander in Einklang zu bringen sind. Im Rahmen der Compliance, wo Untersuchungsverfahren durchgeführt werden, geht es in der heutigen Diskussion zum Beispiel um die Frage, inwiefern die Beweise eines solchen »Compliance-Untersuchungsverfahrens « überhaupt als Beweise im Strafverfahren dienen können, was uns zu der praktischen Dimension der Norm bringt: Hier handelt es sich um einen Fall, wo die Normen, die »einige« betreffen, zu Schwierigkeiten bei der Anwendung von Normen führen können, die »alle« betreffen.

\section{Fazit}

Obwohl es für ein abschließendes Fazit im Zusammenhang mit dem dargestellten Problem verfrüht ist, lassen sich die wichtigsten Schlussfolgerungen dieses Beitrags wie folgt zusammenfassen: 
- Der Zusammenschluss zu einer Gemeinschaft ist eine Voraussetzung für den Bedarf an Normen.

- Normen können nur einem kollektiven Willensakt entsprechen, wenn sie von einem Normgeber mit auctoritas genehmigt wurden.

- Die Norm hat ihren Anwendungsort im Kollektiv.

- Normadressaten können nur Mitglieder einer Gemeinschaft sein.

Die Wechselwirkung von Kollektivierung und Kollektiv stellt für das Strafrecht die Frage nach dem normativen Gehalt von Tatsachen - die Kompatibilisierung von unterschiedlichen Handlungsmustern - und die Frage von übereinander und gegeneinander stehenden Normen und deren Adressaten. 
